\title{
Performance Evaluation of a Handover Scheme for Fast Moving Objects in Hierarchical Mobile Networks
}

\author{
In-Hye Shin ${ }^{*}$, Gyung-Leen Park ${ }^{* *}$, and Junghoon Lee \\ Department of Computer Science and Statistics, \\ Cheju National University, Jeju, Korea \\ \{ihshin76, glpark, jhlee\}@cheju.ac.kr
}

\begin{abstract}
Reducing the handover latency has been one of the most critical research issues in Mobile IPv6. The research includes the fast handover, the hierarchical handover, and variations of them. This paper proposes a variation of the hierarchical handover and develops analytical models to compare the performance of the proposed scheme with that used in the hierarchical mobile networks. The performance evaluation shows that the proposed scheme is very effective for applications like Telematics where mobile nodes move fast. The paper also gives readers the threshold values with which they can select an optimal handover scheme for the given applications.
\end{abstract}

\section{Introduction}

The mobility support in Mobile IPv6 [1] is one of the most important research issues to provide many mobile users with the seamless Internet services. In the Internet environments, when a Mobile Node (MN) moves and attaches itself to another network, it needs to obtain a new IP address. Mobile IPv6 describes how the MN maintains connectivity to the Internet when it changes its Access Router (AR) into another. The process is called handover. During this process, there is a time period when the MN is unable to send or receive the IPv6 packets due to link switching delay and IP protocol operations. The time period is called handover latency. Reducing the handover latency has been a critical research issue to support the seamless service for mobile users. There have been many standardization works such as the hierarchical Mobile IPv6 mobility management (HMIPv6) [2], [3], the fast handover for Mobile IPv6 (FMIPv6) [3], [4], and the simultaneous bindings for fast handover [5]. The paper focuses on the handover in the hierarchical mobile networks. We propose a variation, which can be adapted to applications like Telematices where MNs move fast.

The rest of the paper is organized as follows. Section 2 proposes a new handover scheme. Section 3 presents the analytical models and shows the result of the performance evaluation. Finally, Section 4 concludes the paper.

This research was supported by the MIC(Ministry of Information and Communication), Korea, under the ITRC support program supervised by the IITA (IITA-2005-C1090-05020009.

** Corresponding author. 


\section{The Proposed Approach}

Figure 1 describes the basic operation of the proposed handover scheme. The main idea of the proposed scheme is that it carries out the Mobility Anchor Point (MAP) binding with an MN's Home Address instead of its Regional Care-of Address (RCoA). When an MN enters a new MAP domain as shown in Figure 1, it discovers a new MAP domain as it receives the MAP Option advertised by the MAP. The MAP informs the visiting MNs of its presence by sending the Router Advertisement (RA) message including its prefix information [7]. And then MN needs to configure only on-Link Care-of Address (LCoA), by appending its interface identifier to the prefix sent by the RA message [6], without configuring a new RCoA when it moves into the MAP domain. The MN initializes the MAP registration with its LCoA and its Home Address. The LCoA is used as the source address of the Binding Update (BU) message as done in the HMIPv6. The Home Address is included in the Home Address Option. The MAP will bind the LCoA to the Home Address instead of the RCoA. Thus, the proposed scheme does not have to perform the Duplicate Address Detection (DAD) process for the RCoA any more. Note the fact that the MN's Home address replaces its RCoA. The MAP may include an On-link Care-Of address Test (OCOT) Option in the Binding Acknowledgement (BA). The OCOT Option is in detail specified in [2]. The last process is identical as done in the HMIPv6.

After the MAP registeration, the MN must register its MAP's IP address, included in the MAP Option, with its HA or CNs by sending a BU message that specifies the binding of the MAP's IP address and its home address. The home address is put in the Home Address Option and the MAP's IP address can be involved in the source address field or the Alternate Care-of Address Option. The IP address of the MAP is used instead of the RCoA.

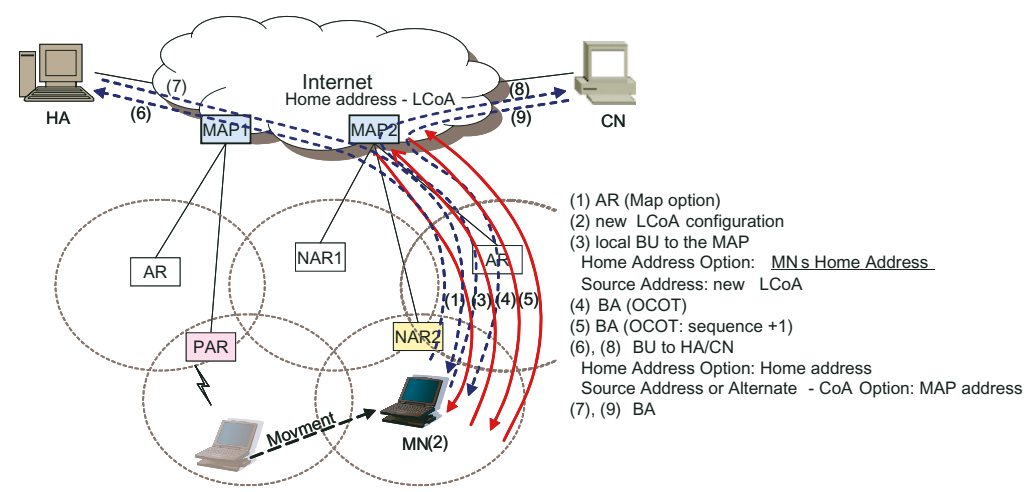

Fig. 1. The Handover Process Supporting Macro Mobility in the Proposed Scheme

The proposed scheme deletes the overhead of configuring the RCoA and the corresponding DAD procedure while adding the overhead of the home address in the data packet. Thus, the proposed scheme will be appropriate for the application like Telematics where a mobile user moves very fast across MAP domains. 


\section{Performance Evaluation}

Table 1 depicts the notations used in the analytical models. The default MTU size for IPv6 packets on an Ethernet is 1500 bytes [8]. The delay for the DAD is set to the MAX_RTX_SOLICITION_DELAY (1 second), the maximum transmission delay of the NS message [7]. The total of the packet size includes the IPv6 Basic Header and some of optional IPv6 Extension Headers. The other IPv6 Extension Headers are omitted due to the variety of its size and the optional preference.

Table 1. The Notations Used in the Model

\begin{tabular}{|c|l|l|}
\hline Notation & Description & Value \\
\hline$T_{s i m}$ & The Total Simulation Time (min) & 15 \\
\hline$V_{M N}$ & The MN's Speed (km/min) & 1 \\
\hline$S_{M A P}$ & The Size of MAP (km) & $1 \sim 16$ \\
\hline$N_{M A P}$ & The total number of Map Update : $\left\lfloor\left(T_{\text {sim }} \times V_{M N}\right) / S_{M A P}\right\rfloor$ & \\
\hline$p$ & The Frequency of Packet Transmission $(1 / \mathrm{min})$ & $1 \sim 30$ \\
\hline$N_{p k t}$ & The Total number of Packet Transmission & \\
\hline$V_{N e t}$ & $\begin{array}{l}\text { The Network Transfer Speed (byte per second) } \\
:\{50(\text { Kbps) } \times 1024\} / 8=6400\end{array}$ & 6400 \\
\hline$D_{\text {Net }}$ & The Network Delay per MTU (s) : $p \times M T U / V_{\text {Net }}$ & \\
\hline$M T U$ & The Size of MTU (byte) & 1500 \\
\hline Data & The Size of Transfer Data (byte) & 40 \\
\hline$I P H$ & The Size of IP Header (byte) [8] & \\
\hline$I P E H$ & The Size of IP Extension Header (byte) [8] & 20 \\
\hline Auth & The Size of Authentication Header (byte) [9] & 20 \\
\hline DestOp & The Size of Destination Options Header (byte) [8] & 8 \\
\hline Frag & The Size of Fragment Header (byte) [8] & 1 \\
\hline$D_{\text {DAD }}$ & The Delay for DAD (s) & \\
\hline HMIPv6 & The Delay using the Hierarchical Mobile IPv6 during $S_{M A P}(\mathrm{~s})$ & \\
\hline proposed & The Delay using the proposed scheme during $S_{M A P}(\mathrm{~s})$ & \\
\hline
\end{tabular}

The following HMIPv6 and proposed represent the additional delay for the hierarchical handover and that for the proposed scheme, respectively.

$$
\begin{gathered}
H M I P v 6=N_{M A P} \times D_{D A D}+\left\lceil\frac{\text { Data }}{M T U-\{I P H+I P E H(\text { Auth }+ \text { Frag })\}}\right\rceil \times T_{\text {sim }} \times D_{\text {Net }} \\
\text { proposed }=\left\lceil\frac{\text { Data }}{M T U-\{I P H+I P E H(\text { Auth }+ \text { Frag }+ \text { DestOp })\}}\right\rceil \times T_{\text {sim }} \times D_{\text {Net }}
\end{gathered}
$$

Figure 2-(a) shows that the larger the size of MAP is, the smaller the difference between two schemes will become. Figure 2-(b) shows that the delay of the proposed scheme is less than that of HMIPv6 at the same rate. The difference results from the DAD cost and is small in this case. Figure 2-(c) and Figure 2-(d) provide the threshold value in the specific point, respectively. 

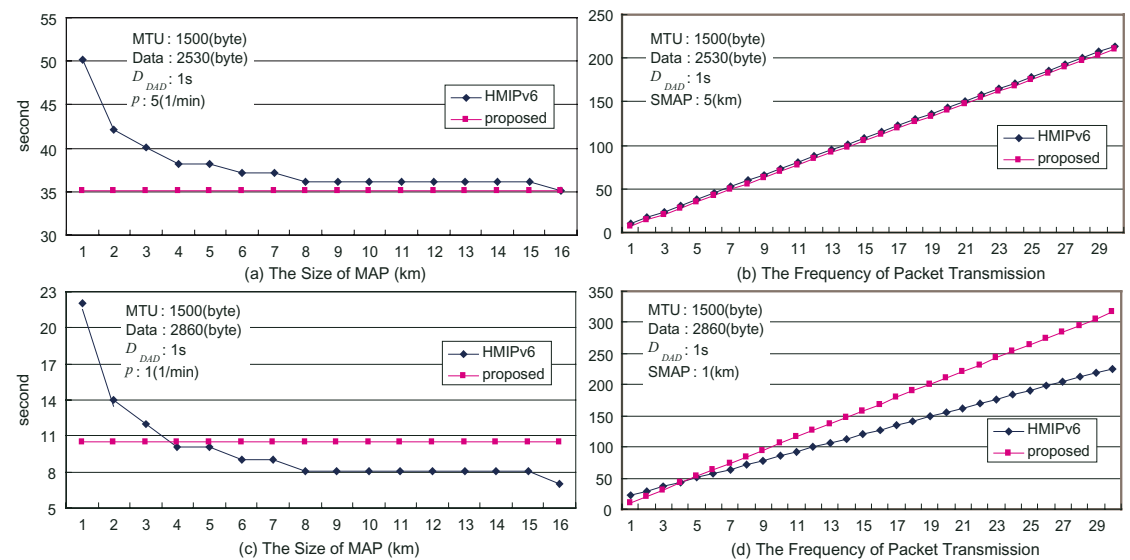

(b) The Frequency of Packet Transmission

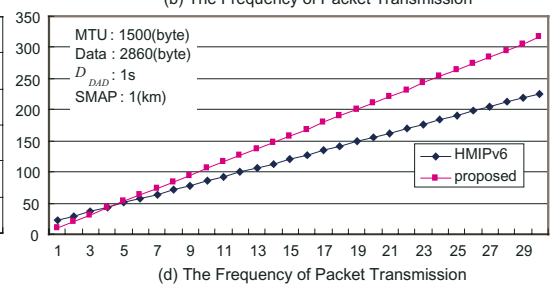

Fig. 2. The Delay according to the Size of the MAP and the Frequency of Packet Transmission

\section{Conclusion}

The paper proposes a variation of the handover scheme used in the HMIPv6. The paper also develops the analytical models to compare the performance of the proposed scheme and that used in the HMIPv6. The result shows that the proposed scheme is very effective regardless of the frequency of the packet transmission if it enables the packet to transfer by a MTU and the MN moves fast. In particular, the proposed scheme can be adapted to Telematics services where an user drives fast and sometimes receives the Internet services using the terminal in the car.

\section{References}

1. Johnson, D., Perkins, C., Arkko, J.: Mobility Support in IPv6. IETF RFC 3775 (2004)

2. Soliman, H., Castelluccia, C., Malki, K., Bellier, L.: Hierarchical Mobile IPv6 Mobility Management (HMIPv6). Internet Draft, IETF, draft-ietf-mobileip-hmipv6-08.text (2003)

3. Jung, H., Koh, S.: Fast Handover Support in Hierarchical Mobile IPv6. IEEE Conference Proceeding, Vol. 2. (2004) 551-554

4. Koodli, R.: Fast Handovers for Mobile IPv6. IETF RFC 4068 (2005)

5. Hsieh et al., R.: S-MIP: A Seamless Handoff Architecture for Mobile IP. in Proc. INFOCOM 2003 (2003)

6. Thomson S., Narten, T.: IPv6 Stateless Address Configuration. IETF RFC 2462 (1998)

7. Narton, T., Nordmark, E., Simpson, W.: Neighbor Discovery for IP Version 6 (IPv6). IETF RFC 2461 (1998)

8. Deering, S., Hinden, R.: Internet Protocol, Version 6 Specification. IETF RFC 2460 (1998)

9. Kent, S., Atkinson,R.: IP Authentication Header. IETF RFC 2402 (1998). 